\title{
Characterization of Low-mass K2 Planet Hosts Using Near-infrared Spectroscopy
}

Martínez, Romy Rodríguez; Ballard, Sarah; Mayo, Andrew; Vanderburg, Andrew; Montet, Benjamin T.; Christiansen, Jessie L.

Published in:

Astrophysical Journal

Link to article, DOI:

$10.3847 / 1538-3881 / a b 3347$

Publication date:

2019

Document Version

Publisher's PDF, also known as Version of record

Link back to DTU Orbit

Citation (APA):

Martínez, R. R., Ballard, S., Mayo, A., Vanderburg, A., Montet, B. T., \& Christiansen, J. L. (2019).

Characterization of Low-mass K2 Planet Hosts Using Near-infrared Spectroscopy. Astrophysical Journal, 158(4), [135]. https://doi.org/10.3847/1538-3881/ab3347

\section{General rights}

Copyright and moral rights for the publications made accessible in the public portal are retained by the authors and/or other copyright owners and it is a condition of accessing publications that users recognise and abide by the legal requirements associated with these rights.

- Users may download and print one copy of any publication from the public portal for the purpose of private study or research.

- You may not further distribute the material or use it for any profit-making activity or commercial gain

- You may freely distribute the URL identifying the publication in the public portal 


\title{
Characterization of Low-mass K2 Planet Hosts Using Near-infrared Spectroscopy
}

\author{
Romy Rodríguez Martínez ${ }^{1}$, Sarah Ballard ${ }^{2,9}$, Andrew Mayo ${ }^{3,4,5,10,11}$ (i) , Andrew Vanderburg ${ }^{6,12}$ (i), Benjamin T. Montet ${ }^{7,12}$ (1), and \\ Jessie L. Christiansen ${ }^{8}$ (D) \\ ${ }^{1}$ Department of Astronomy, The Ohio State University, 140 West, 18th Avenue, Columbus, OH 43210, USA; rodriguezmartinez.2@osu.edu \\ ${ }^{2}$ MIT Kavli Institute for Astrophysics \& Space Research, Cambridge, MA 02139, USA \\ ${ }^{3}$ Astronomy Department, University of California Berkeley, Berkeley, CA 94720-3411, USA \\ ${ }^{4}$ DTU Space, National Space Institute, Technical University of Denmark, Elektrovej 327, DK-2800 Lyngby, Denmark \\ ${ }^{5}$ Centre for Star and Planet Formation, Natural History Museum of Denmark \& Niels Bohr Institute, University of Copenhagen, Øster Voldgade 5-7, DK-1350 \\ Copenhagen K., Denmark \\ ${ }^{6}$ Department of Astronomy, The University of Texas at Austin, Austin, TX 78712, USA \\ ${ }^{7}$ Department of Astronomy and Astrophysics, University of Chicago, 5640, S. Ellis Avenue, Chicago, IL 60637, USA \\ ${ }^{8}$ NASA Exoplanet Science Institute, California Institute of Technology, M/S 100-22, 770 S. Wilson Avenue, Pasadena, CA, USA \\ Received 2018 August 8; revised 2019 July 15; accepted 2019 July 16; published 2019 September 6
}

\begin{abstract}
We present moderate resolution near-infrared spectra in the $H, J$, and $K$ band of M-dwarf hosts to candidate transiting exoplanets discovered by NASA's $K 2$ mission. We employ known empirical relationships between spectral features and physical stellar properties to measure the effective temperature, radius, metallicity, and luminosity of our sample. Out of an initial sample of 56 late-type stars in $K 2$, we identify 35 objects as $\mathrm{M}$ dwarfs. For that subsample, we derive temperatures ranging from 2870 to $4187 \mathrm{~K}$, radii of $0.09-0.83 R_{\odot}$, luminosities of $-2.67<\log L / L_{\odot}<-0.67$, and $[\mathrm{Fe} / \mathrm{H}]$ metallicities between -0.49 and 0.51 dex. We then employ the stellar properties derived from spectra, in tandem with the $K 2$ light curves, to characterize their planets. We report 33 exoplanet candidates with orbital periods ranging from 0.19 to 21.16 days, and median radii and equilibrium temperatures of $2.3 R_{\oplus}$ and $986 \mathrm{~K}$, respectively. Using planet mass-radius relationships from the literature, we identify seven exoplanets as potentially rocky, although we conclude that probably none reside in the habitable zone of their parent stars.
\end{abstract}

Key words: planetary systems - planets and satellites: fundamental parameters - stars: fundamental parameters stars: late-type - stars: low-mass - techniques: spectroscopic

\section{Introduction}

Since its launch in 2009, the NASA Kepler spacecraft has gathered exquisite photometry of over 150,000 stars and has uncovered thousands of exoplanets in our galaxy via the transit photometry method (Borucki et al. 2010, 2011; Batalha et al. 2013; Burke et al. 2014). Kepler continuously monitored the same part of the sky for four years, until reaction wheel failure compromised the pointing stability of the spacecraft. However, engineers soon found a way to balance the spacecraft using solar pressure and repurposed it for a new mission, $K 2$ (Howell et al. 2014). In this new mode of operation, $K 2$ observes different regions along the ecliptic, targeting between 10 and 30 thousand stars for approximately 80 days. $K 2$ is therefore particularly suited for searches of transiting exoplanets in shortperiod orbits.

The motivations for targeting $\mathrm{M}$ dwarfs for both exoplanet searches and follow-up observations are manifold. First, M dwarfs are the most common type of star, comprising nearly $70 \%$ of all stars in the Milky Way (Bochanski et al. 2010). Second, although they were initially thought to host planets infrequently for their dearth of Jupiter-sized planets, the Kepler and $K 2$ missions revealed that $\mathrm{M}$ dwarfs form smaller (potentially rocky) planets in greatest abundance (Howard et al. 2012). Studies have shown that for planets with periods of less than 50 days, planets between 2 and $4 R_{\oplus}$ are twice as

\footnotetext{
${ }^{9}$ MIT Torres Fellow.

${ }^{10}$ National Science Foundation Graduate Research Fellow.

${ }^{11}$ Fulbright Fellow.

12 NASA Sagan Fellow.
}

abundant around $\mathrm{M}$ dwarfs than around Sun-like stars (Howard et al. 2012; Mulders et al. 2015). Dressing \& Charbonneau (2015) found that the mean number of small planets $\left(0.5-4 R_{\oplus}\right)$ per late $\mathrm{K}$ dwarf or early $\mathrm{M}$ dwarf is $2.5 \pm 0.2$ for orbital periods shorter than 200 days, comparable to the $2.0 \pm 0.45$ determined by Mulders et al. (2014). This fact, combined with the ubiquity of $\mathrm{M}$ dwarfs, establish them as the majority of hosts to small planets in the Milky Way. Moreover, stars cooler than $4000 \mathrm{~K}$ make up 25\% of the Transiting Exoplanet Survey Satellite (TESS) Input Catalog (Sullivan et al. 2015; Muirhead et al. 2018), as opposed to 5\% of the Kepler Input Catalog (Brown et al. 2011). Additionally, the smaller radii and masses of M dwarfs translate to larger transit depths, larger radial velocity semi-amplitudes, and larger transmission spectroscopy signals for exoplanet study (for a detailed summary of the advantages and complications of $\mathrm{M}$ dwarfs as planet host stars, see Shields et al. 2016). The recent discoveries of small, temperate exoplanets circling $\mathrm{M}$ dwarfs, such as Proxima $\mathrm{b}$ (Anglada-Escudé et al. 2016), the TRAPPIST-1 system (Gillon et al. 2017), and LHS1140 b (Dittmann et al. 2017), have further demonstrated the feasibility of targeting cool, small stars in the search for potentially life-bearing worlds.

Despite these facts, there is a relative paucity of detected planets orbiting $\mathrm{M}$ dwarfs. They number several hundred, as compared to the several thousand of their FGK counterparts. ${ }^{13}$ They are challenging to characterize from spectra (Torres et al. 2011, with summary in Shields et al. 2016) and also comprised a small fraction in the Kepler Input Catalog (Brown et al. 2011).

$\overline{13}$ https://exoplanetarchive.ipac.caltech.edu/ 
However, recent studies have made critical inroads linking spectral features to physical properties of $\mathrm{M}$ dwarfs (Boyajian et al. 2012; Mann et al. 2012, 2013b; Rojas-Ayala et al. 2012; Terrien et al. 2012; Newton et al. 2015).

Precise stellar characterization is ultimately crucial to understand the planet sample. The characteristics of these new worlds are so closely tied to the physical properties of their host stars that we must understand the stars first if we aim to understand the planets in detail. Eking out the mass-radius relationship of exoplanets, for example, relies on large spectroscopic or asteroseismic surveys to characterize the host stars to better than 10\% (Huber et al. 2013; Weiss et al. 2013; Dressing et al. 2014; Wolfgang et al. 2016; Fulton et al. 2017; Van Eylen et al. 2018). Furthermore, the deluge of exoplanet discoveries and our limited resources make it impossible to follow-up every single planet candidate. Reliably identifying the most promising candidates for follow-up characterization demands that we know the characteristics of the candidates.

For $K 2$, in contrast to Kepler, the target selection has been proposal driven. Likewise, stellar characterization of large samples of $K 2$ planet host stars has been an ongoing community effort (Huber et al. 2016; Dressing et al. 2017a). To contribute to this endeavor, we present in this study the stellar characterization of 35 candidate exoplanet host stars from $K 2$ with near-infrared spectra.

We infer the temperatures, radii, luminosities, masses, and metallicities of the stellar sample using empirial relationships. We subsequently estimate the radii and equilibrium temperatures of the planet candidates. The paper is organized as follows. In Section 2, we describe the observation techniques and the reduction pipeline of our spectra. In Section 3, we present an analysis of the data, the derivation of the equivalent widths (EWs) of different metals, and a comparison of our derived EWs of the aluminum feature at 1.67 microns to those previously published. In Section 4, we summarize the results of our analysis for the cool dwarf sample. In Sections 5 and 6, we explain the derivation of the planet parameters and discuss the potential habitability of the planets. We identify two systems suitable for follow-up Doppler and atmospheric characterization, and we highlight several false positives from the $K 2$ photometry pipeline. In Section 7, we conclude and summarize our findings and recommendations for follow-up observations.

\section{Observations}

We gathered our spectra with the near-infrared TripleSpec spectrograph (Wilson et al. 2004) at the Palomar $5 \mathrm{~m}$ telescope. The TripleSpec instrument has a $1^{\prime \prime} \times 30^{\prime \prime}$ slit, a moderate resolution of $R=2500-2700$, and a wavelength coverage in the near-infrared of 1.0-2.4 $\mu \mathrm{m}$. We selected our sample of 56 late-type stars identified as potential planet hosts from the first two years of the $K 2$ mission. These targets were selected based on standard observing constraints, specifically, with the aim of minimizing airmass, avoiding clouds, and focused preferentially on the bright targets. For each science target, we gathered observations in an ABBA nod sequence, with exposure times between 5 and $300 \mathrm{~s}$. We also gathered spectra of telluric standards at hourly intervals. We used the bright quartz lamp to gather flat fields at the beginning and end of each night, in addition to collecting dark frames.

To reduce our spectra, we used Spextool, a publicly available IDL-based package for spectral reduction (Cushing et al. 2004). For correction of telluric lines, we used xtellcor
(Vacca et al. 2003). When choosing a spectral line in the A0 star that is unaffected by atmospheric absorption, we selected the Paschen $\delta$ line at $1.005 \mu \mathrm{m}$, following the TripleSpec manual suggestions. This spectral line is used to constrain the kernel in xtellcor.

The observations were carried out during the nights of UT 2016 January 21 and UT 2016 January 22. The seeing fluctuated between 1.5 and 1 ".7. The observing conditions varied slightly, from mostly good throughout the first half of the night, followed by cirrus clouds dominating the observations. During the second night, the atmospheric variability coupled with long exposures to compensate for the poor weather began affecting the observations to the point where some of the spectra were too low signal-to-noise to yield reliable estimates of the stellar parameters, or the spectra could not even be reduced at all. We therefore removed all those objects along with targets with exposures of $300 \mathrm{~s}$ or more, as the sky was too variable over that duration, making the sky subtraction challenging. These targets are: EPIC 211432922, EPIC 211694226, EPIC 210512752, EPIC 210625740, EPIC 210625740, EPIC 212092746, EPIC 212152341, EPIC 212069861, EPIC 211946007, EPIC 212152341, EPIC 211991987, and EPIC 201247497.

We also rejected all the reduced stars with derived temperatures below $\sim 2800 \mathrm{~K}$ or above $4800 \mathrm{~K}$ since those are outside the range for which the empirical relationships for cool dwarfs used here are valid. ${ }^{14}$ In particular, we rejected the targets EPIC 210769880, EPIC 211694226, and EPIC 201155177.

\subsection{Sample Contamination by Other Spectral Types}

One of the challenges in characterizing $M$ dwarfs from Kepler and $K 2$ samples is contamination by red giants. The target selection process for $K 2$ is complex and varies between different proposing teams, but many proposals were based on color and proper motion cuts, since spectra and parallaxes were not available for most stars at the time of the proposal selection. This implies that many samples of putative low-mass red stars are polluted by red giants or hotter stars reddened by interstellar extinction. Mann et al. (2012) found that, from a sample of 382 supposed $\mathrm{M}$ dwarfs from Kepler, the majority were in fact giants. This suggests that the identification of $\mathbf{M}$ dwarfs from photometry alone in large samples is unreliable or impossible without spectroscopic follow-up. We therefore began our analysis of the sample by visually inspecting all of the spectra to eliminate the red giants or stars with other classifications. We compared features that appear sharply distinct in red giants and red dwarfs. Some of the most prominent relative differences in the $J, H$, and $K$ bandpasses in dwarfs and giants are in the lines $\operatorname{Mg}(1.50 \mu \mathrm{m}), \operatorname{Mg}(1.71 \mu \mathrm{m})$ and $\mathrm{Na}$ at $2.2 \mu \mathrm{m}$. The results of this analysis show that, from a total of 56 red stars, only 35 are consistent with M-dwarf classification, while the remaining 21 were either (1) not usable/discarded for the reasons summarized in the previous section, or (2) their spectral features were inconsistent with those of $\mathrm{M}$ dwarfs, although we do not

\footnotetext{
14 The Newton et al. (2015) relations are appropriate for stars between 3200 and $4800 \mathrm{~K}$. For four of our stars, after employing the metrics from Newton et al. (2015), we find mean temperatures that lie below this prescribed range (EPIC 210564155, EPIC 211817229, EPIC 210659688, EPIC 212092746). We include their temperatures in Table 1, but compare them with those reported in Dressing et al. (2017a). We find that the temperatures we determine are generally consistent with that work, suggesting that the relationships may be applicable for stars as cool as $2800 \mathrm{~K}$.
} 
attempt to classify those. Some of those stars include EPIC 210769880, classified as a $K 2$ giant in Dressing et al. (2017a), EPIC 211762841, a K7 dwarf as classified in the same work, and EPIC 211822797, another K7 dwarf. Finally, EPIC 211694226 is classified in Dressing et al. (2017a) as an M3 dwarf with a nearby companion which may or may not be physically associated. When reducing the spectrum of this star, the light from the companion contaminated it and we could not properly reduce it so we exclude it from the characterization.

In addition to visually inspecting the spectra and comparing several features of interest between red dwarfs and giants, we also supported our M-dwarf classification with newly available distances inferred from Gaia DR2 parallaxes. We found that the sources with spectra consistent with M-dwarf classification also had distances close enough to be $\mathbf{M}$ dwarfs rather than giants. In particular, the distances from Gaia for these objects range from 43 to $497 \mathrm{pc}$.

\section{Analysis}

To extract the physical properties of $M$ dwarfs from their spectra, we cannot directly compare whole synthetic spectra to observed spectra as for FGK dwarfs (see the review of Shields et al. 2016 for a detailed summary). Current best practices involve empirical relationships between physical properties and spectral features, painstakingly acquired with benchmark binary systems or interferometric measurements. With the exception of Mann et al. (2013a), in which the authors identified large sections in which synthetic spectra do reliably replicate observed spectra, most published relationships employ spectral indices and EWs of absorption features across the optical and near-infrared (Mann et al. 2012; Rojas-Ayala et al. 2012; Terrien et al. 2012; Newton et al. 2015). We note that the EW of a spectral feature is defined as

$$
\mathrm{EW}_{\lambda}=\int_{\lambda_{1}}^{\lambda_{2}}\left[1-\frac{F(\lambda)}{F_{c}(\lambda)}\right] d \lambda
$$

where $F(\lambda)$ is the flux of the absorption line integrated between $\lambda_{1}$ and $\lambda_{2}$ and $F_{c}(\lambda)$ is the continuum flux.

We elect to employ here the relationships of Newton et al. (2015) to derive stellar radius from the spectrum, and Mann et al. (2013b) for deriving metallicity. We selected the metrics with smallest intrinsic scatter, which are $H$ band from Newton et al. (2015) for stellar radius and effective temperature, and $K$ band from Mann et al. (2013b) for metallicity. The relationships from Newton et al. (2015) are generally applicable to spectral types between mid-K and mid-M, with radii of $0.18<R / R_{\odot}<0.8$, temperatures of $3200 \mathrm{~K}<T_{\text {eff }}<$ $4800 \mathrm{~K}$, and $\log$ luminosities of $-2.5<\log \left(L / L_{\odot}\right)<-0.5$.

In that work, they determined that the stellar effective temperature correlated most strongly with the EWs of the aluminum doublet at $1.67 \mu \mathrm{m}$ and magnesium absorption at $1.50 \mu \mathrm{m}$, with intrinsic scatter of $73 \mathrm{~K}$. The same aluminum doublet, in addition to magnesium absorption at $1.57 \mu \mathrm{m}$, traced the stellar radius, with intrinsic scatter of $0.027 R_{\odot}$. We also measure stellar luminosity, which Newton et al. (2015) found to be correlated with the EWs of magnesium features at 1.50 and $1.71 \mu \mathrm{m}$, with intrinsic scatter of 0.049 in $\log \left(L / L_{\odot}\right)$.

These relationships were calibrated from the Infrared Telescope Facility (IRTF)/SpeX spectra, which have a lower resolution than the TripleSpec spectra used in this work. Dressing et al. (2017a) also used TripleSpec and downgraded
Rodriguez et al. 2018 - Dressing et al. 2017

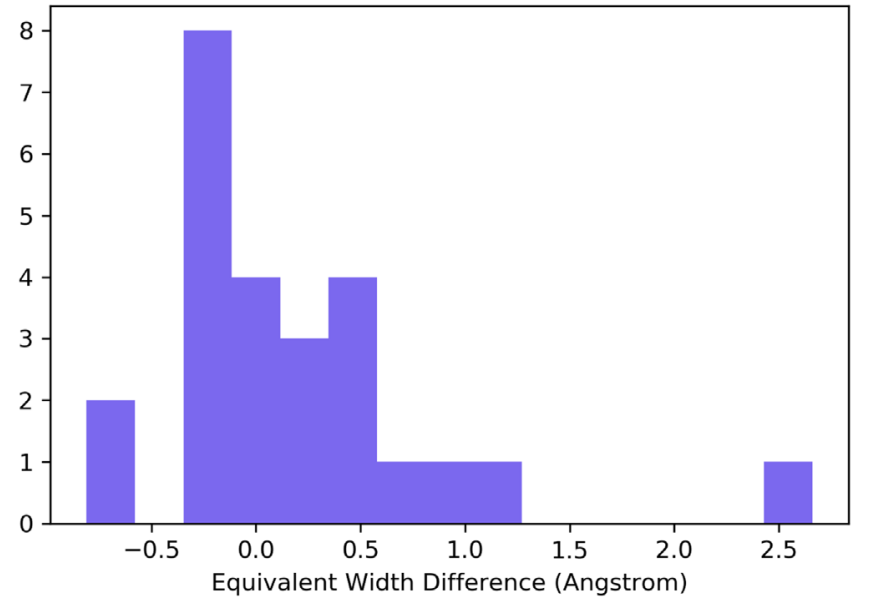

Figure 1. Difference between two distributions: the EWs of the line $\mathrm{Mg}$ $(1.57 \mu \mathrm{m}$; which is sensitive to the stellar radius) of the cool dwarfs in this paper and the EWs of that same spectral line for those same stars previously characterized in Dressing et al. (2017a). The outlier on the right is EPIC 210508766, which has a slightly higher than average radius uncertainty.

their spectra to match the resolution of IRTF/SpeX. They identified a systematic offset in EW of $0.1 \AA$, depending upon whether the Newton et al. (2015) relations were applied to spectra from the TripleSpec or SpeX spectrographs. We find a typical uncertainty of $\sim 20 \%$ on individual EWs from our spectra, all gathered with TripleSpec (as we show in Figure 1). We conclude that the contribution to the error budget from the Poisson uncertainty is larger by a factor of 2-3 than this systematic offset, and we do not include it.

Mann et al. (2013a) also used the EWs of absorption features to determine the $[\mathrm{Fe} / \mathrm{H}]$ and $[\mathrm{M} / \mathrm{H}]$ metallicity of cool dwarfs. They found that the lines most sensitive to $[\mathrm{Fe} / \mathrm{H}]$ metallicity are features in the $K$ band, including $\mathrm{Na}$ at $2.2 \mu \mathrm{m}$. The metallicity measurement technique presented in Mann et al. (2013a) employs the temperature-sensitive $\mathrm{H}_{2} \mathrm{O}-\mathrm{K} 2$ index introduced by RojasAyala et al. (2012), which measures the deformation of the spectrum in the $K$ band due to water absorption, and is temperature sensitive. Because the index saturates at temperatures close to $4000 \mathrm{~K}$, the metallicity relations from Mann et al. (2013a) are not reliable for hotter stars.

We went on to calculate stellar mass using another empirical relation reported in Mann et al. (2013b). This relation is a thirddegree polynomial with effective temperature. We use the newly derived effective temperature from the Newton et al. (2015) metric to then obtain the stellar mass.

Mann et al. (2013b) caution that these relationships for single stars give increasing errors for stars with $T_{\text {eff }}<3300 \mathrm{~K}$. Because there are several stars below this temperature in our sample, some of the stellar masses yielded negative values as they had very low effective temperatures. For the stars for which the Mann et al. (2013b) relationships yielded negative masses, we employed the mass-radius empirical relationships for single stars (Equation (10)) from Boyajian et al. (2012). For comparison, we estimated the masses of our stars using the model-independent metric from Benedict et al. (2016), which is valid for stars in the range of $0.008<M\left[M_{\odot}\right]<0.6$. We found that they are generally consistent with the Mann et al. (2013b) and the Boyajian et al. (2012) masses to within $\sim 0.2 M_{\odot}$.

Before computing the EWs, we first estimated the continuum by selecting a region to the left and to the right (blueward and 
redward) of the spectral feature of interest (both the line centers, and the regions on either side, are listed in Table 1 of Newton et al. 2015 and Table 5 of Mann et al. 2013a). We then fit a line to the continuum regions on either side of the feature of interest and then divided by this line to normalize the spectrum. To calculate the EWs, we numerically integrated the flux within the absorption feature, and we define the EWs in Angstrom. The derived values for the EWs and masses are shown in Table 2. We tested the numerical approach of measuring EWs by fitting Gaussian curves to the absorption lines with Gaussian profiles. The values obtained from both methods are in good agreement, though we elected to use the numerical method to measure EWs of all the absorption features in this work, as there are many lines with nonGaussian profiles (such as the aluminum doublet at $1.67 \mu \mathrm{m}$ ). Figure 1 depicts the difference between our derived EW values of $\mathrm{Al}-\mathrm{a}(1.67 \mu \mathrm{m})$ for the set of 24 stars in our sample that overlap with Dressing et al. (2017a). We are consistent with that work to within $0.5 \AA$ with $68 \%$ confidence. We observed similar differences between our derived EWs and those from Dressing for all the other relevant absorption features. We found that those differences correspond to differences within $\pm 400 \mathrm{~K}$ in stellar effective temperature and $\pm 0.2 R_{\odot}$ in radii. More generally, our measured EWs have larger error bars on average than those reported in Dressing et al. (2017a), which propagate to larger uncertainties in the resulting stellar parameters. However, these discrepancies can be largely explained by the higher signal-to-noise ratio $(\mathrm{S} / \mathrm{N})$ spectra used in Dressing et al. (2017a) ( $\mathrm{S} / \mathrm{N} \sim 200$ versus $\sim 50-100$ in this work), and the final stellar parameters reported here are consistent with that work within the uncertainties.

We employed a Monte Carlo technique to measure the uncertainties on our EWs and the corresponding stellar parameters. We generated 100 synthetic spectra from each individual spectrum with the same noise properties as the real spectra, then calculated all the parameters of interest in each of them. We then took the mean as the true measured value and the standard deviation as the error associated to each stellar property.

\section{Results}

\subsection{Effective Temperature}

By applying the empirical calibrations from Newton et al. (2015) for temperature, and by the measuring the EWs of the Al-a $(1.67 \mu \mathrm{m})$, Al-b $(1.67 \mu \mathrm{m})$, and $\mathrm{Mg}(1.50 \mu \mathrm{m})$ lines, we estimated the stellar effective temperature for all the stars for which we had reduced spectra. The temperatures for our sample range from 2870 to $4187 \mathrm{~K}$, which brackets spectral types between M5 and K7 (Reid \& Hawley 2005; Boyajian et al. 2012). Figure 2 shows a histogram of the distribution of temperatures of our cool dwarfs. The mean value of the distribution is $3620 \mathrm{~K}$. The median uncertainty in the stellar effective temperature is about $100 \mathrm{~K}$. The error bars in the temperatures of many of the stars are large in part because of the disappearance of the $\mathrm{Mg}$ and $\mathrm{Al}$ lines in the coolest dwarfs, which causes the temperatures to be overestimated (Newton et al. 2015; Dressing et al. 2017a).

\subsection{Stellar Radius}

We estimated the radii of all of the stars in our sample applying the Newton et al. (2015) relationships by calculating
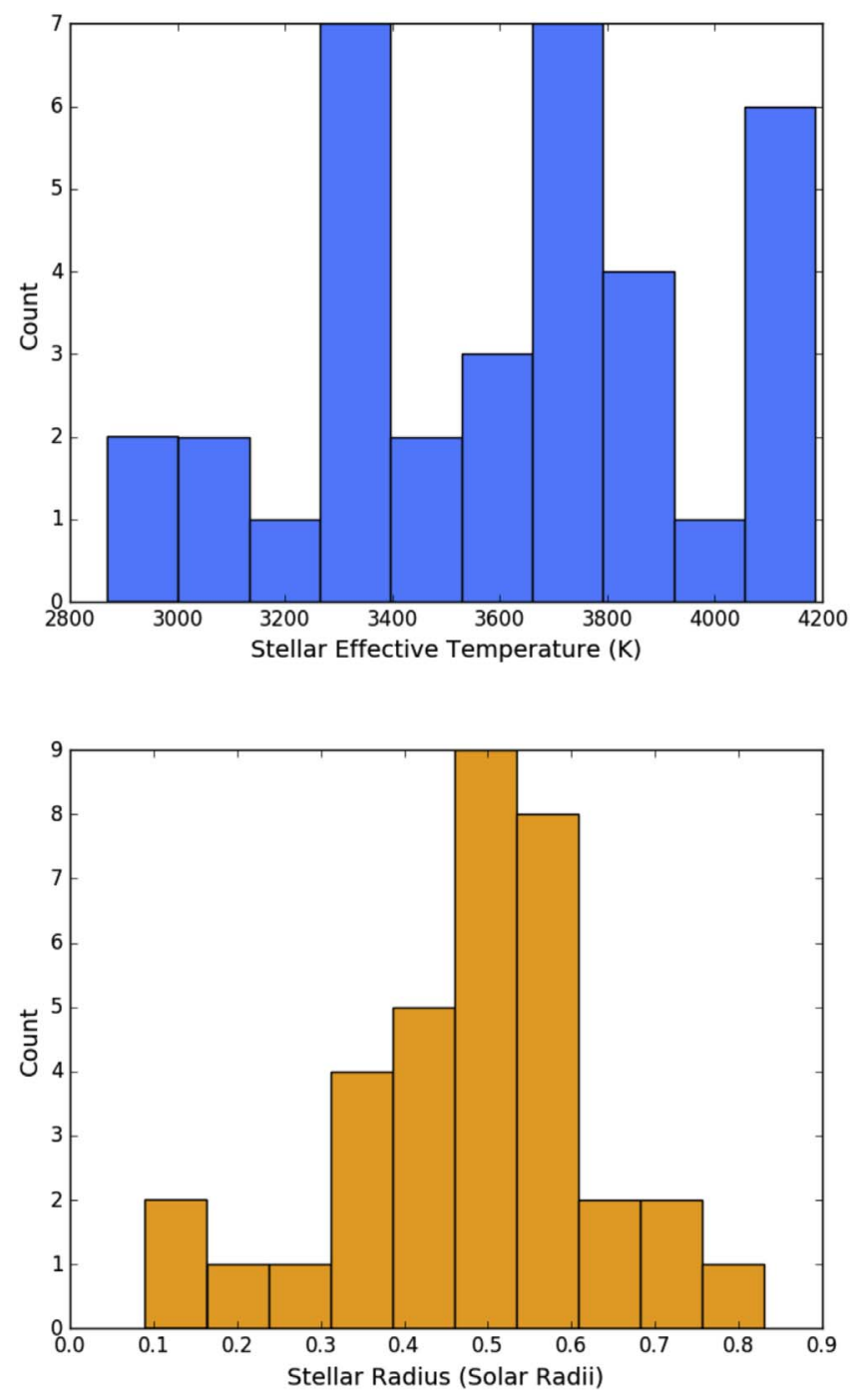

Figure 2. Temperature (top) and radii (bottom) distribution of our stellar sample. The mean temperature is $3681 \mathrm{~K}$ and the mean uncertainty is around $120 \mathrm{~K}$. The radius distribution peaks sharply at $0.48 R_{\odot}$.

the EWs of the sensitive lines to the radius. We obtained a range of radii between $0.09 R_{\odot}$ and $0.83 R_{\odot}$. The mean of the distribution is $0.48 R_{\odot}$. The mean error on our sample is $0.05 R_{\odot}$. Figure 2 shows the radii distribution. As with the temperatures, the range of measured radii is consistent with an M-dwarf classification (see Figure 3 for a scatter plot showing the radius-temperature distribution).

\subsection{Luminosity}

The $\log L / L_{\odot}$ luminosities from our stellar sample were all estimated using the Newton et al. (2015) relations, and yielded a range of luminosities between $-2.67<\log L / L_{\odot}<-0.67$, or between $0.002 L_{\odot}$ and $0.213 L_{\odot}$. Figure 4 shows the range of values for the luminosities. The average log luminosity is -1.59 , and the mean error is 0.13 .

\subsection{Metallicity}

The planet-stellar-metallicity correlation is an active area of research in exoplanets, and previous studies have demonstrated correlations between planet size and mass and stellar 


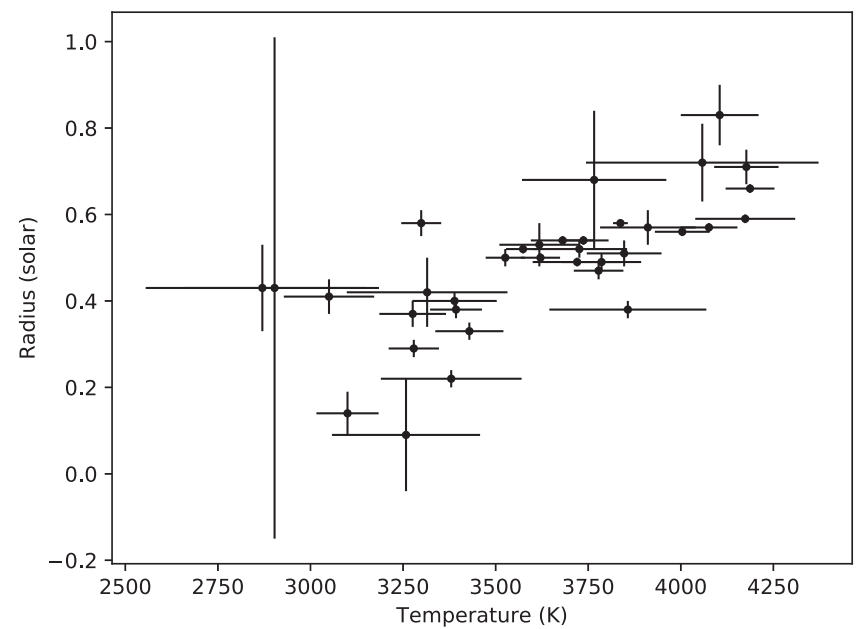

Figure 3. Radius-Temperature scatter plot of our stellar sample.
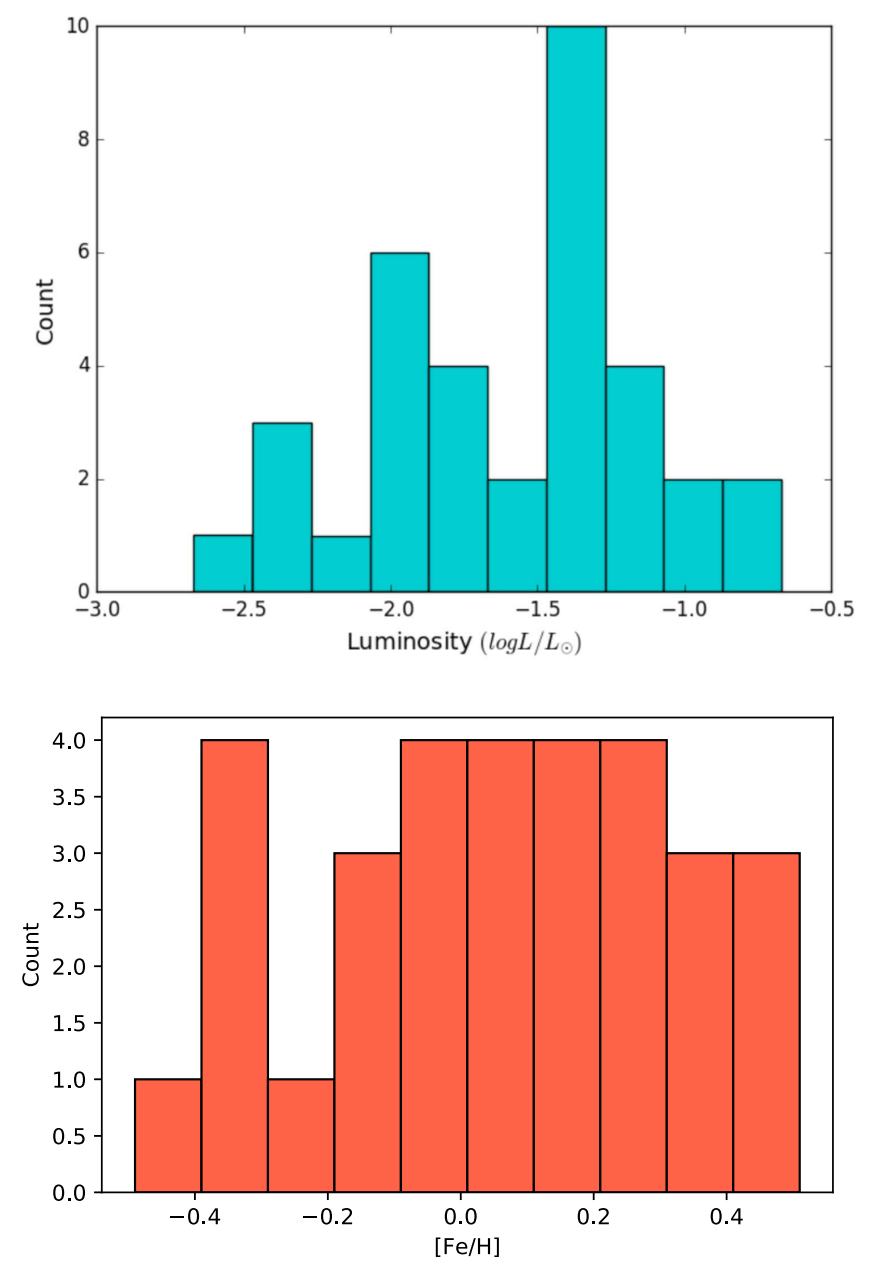

Figure 4. Luminosity (top) and $[\mathrm{Fe} / \mathrm{H}]$ metallicity (bottom) of our sample. The median log luminosity is -1.57 while the mean metallicity is $0.13 \mathrm{dex}$.

metallicities; e.g., hot Jupiters form more frequently around metalrich stars (Santos et al. 2004; Fischer \& Valenti 2005; Johnson \& Apps 2009). Authors in recent years have already hinted at correlations between metallicity and eccentricity (Dawson \& Murray-Clay 2013), and mutual inclinations and planet multiplicity (Ballard \& Johnson 2016). Hirano et al. (2018) found that from a sample of 16 planets orbiting $M$ dwarfs, those larger than $3 R_{\oplus}$ are only found orbiting the most metal-rich hosts.

We derived $[\mathrm{Fe} / \mathrm{H}]$ metallicities in our sample from the Mann et al. (2013a) relationships by calculating the EWs of the features sensitive to metallicity in the $K$ band and by computing the $\mathrm{H}_{2} \mathrm{O}-\mathrm{K} 2$ index in the specified range in that same paper. The metallicities of our sample of $\mathrm{M}$ dwarfs ranged from -0.49 to 0.51 dex. The mean uncertainty in our values is $0.1 \mathrm{dex}$, as can be observed in Figure 4. The range of host star metallicities in our sample is similar to that of surveys of hundreds of nearby M dwarfs from both Rojas-Ayala et al. (2012) and Newton et al. (2014). All the derived physical parameters and uncertainties for our cool dwarfs are listed in Tables 1 and 2.

\subsection{Comparison of Stellar Parameters with Gaia DR2}

We complemented the observations of our sources with the recent second data release of the Gaia mission (Gaia Collaboration et al. 2016, 2018). We corroborated the accuracy of our derived stellar parameters by comparing our luminosities from the Newton et al. (2015) empirical relationships to those predicted by Gaia DR2. First, we computed the absolute magnitude of each star using the exquisitely precise $G$-band magnitudes and distances inferred from the parallaxes delivered by Gaia. We then converted the absolute $G$ magnitudes to bolometric absolute magnitudes by applying the bolometric corrections for cool dwarfs in Jordi et al. (2010). Finally, we converted the bolometric absolute magnitudes to bolometric luminosities and compared them with the luminosities estimated in this work. We found no evidence of offset between the distributions, and conclude that our results are in good agreement with Gaia, as can be observed in Figure 5.

\section{The Planet Sample}

\subsection{Transit Light-curve Reduction and Analysis}

In addition to the stars, we characterized the associated planet candidates using the derived stellar properties presented here. Specifically, we applied the inferred stellar radii, luminosities, and masses to estimate planet radius, equilibrium temperature, and semimajor axis, respectively. We briefly describe the process of reduction and analysis of the $K 2$ light curves of our stellar sample and how we estimated the planet properties.

Before fitting and analyzing the light curves for the transit and planetary parameters, the $K 2$ photometry must be corrected for motion-induced systematics that reduce its photometric precision and introduce noise and artificial variability in the data. We compensate for such instrumental systematics using the reduction strategy outlined in Vanderburg \& Johnson (2014) and later updated in Vanderburg et al. (2016). The first step of this process consists in creating 20 aperture masks of varying sizes and shapes to perform aperture photometry on the $K 2$ targets and produce 20 raw light curves of each. The systematic noise due to the loss of balance and the regular repositioning of the spacecraft is then mitigated essentially by estimating the path of the targets along the CCD and identifying and removing a correlation between Kepler motion and the apparent measured flux. Additionally, we identify and remove the data taken during thruster fires and also eliminate low-frequency $(>0.75$ days) variations. After implementing these steps on all 20 light curves, we pick the aperture mask 
Table 1

Stellar Parameters for $K 2$ Cool Dwarfs

\begin{tabular}{|c|c|c|c|c|c|c|c|c|}
\hline EPIC & $T_{\text {eff }}(\mathrm{K})$ & $\sigma_{T_{\text {eff }}}$ & Radius $\left(R_{\odot}\right)$ & $\sigma_{R}$ & Luminosity $\left(\log \left(L_{\star} / L_{\odot}\right)\right.$ & $\sigma_{\log \left(L_{\star} / L_{\odot}\right)}$ & {$[\mathrm{Fe} / \mathrm{H}](\mathrm{dex})$} & $\sigma_{[\mathrm{Fe} / \mathrm{H}]}$ \\
\hline $210564155^{\dagger}$ & 2870 & 323 & 0.43 & 0.10 & -2.46 & 0.19 & -0.37 & 0.16 \\
\hline $211817229^{\dagger}$ & 2903 & 257 & 0.43 & 0.58 & -2.67 & 0.15 & -0.14 & 0.1 \\
\hline 210659688 & 3050 & 142 & 0.41 & 0.04 & -2.3 & 0.06 & 0.13 & 0.1 \\
\hline 212092746 & 3100 & 111 & 0.14 & 0.05 & -2.03 & 0.07 & 0.27 & 0.15 \\
\hline 211839798 & 3258 & 212 & 0.09 & 0.13 & -2.2 & 0.13 & -0.25 & 0.07 \\
\hline 210931967 & 3276 & 115 & 0.37 & 0.03 & -1.83 & 0.06 & -0.35 & 0.08 \\
\hline $211077024^{\dagger}$ & 3279 & 99 & 0.29 & 0.03 & -1.9 & 0.09 & 0.4 & 0.05 \\
\hline $201205469^{\dagger}$ & 3299 & 90 & 0.58 & 0.04 & -1.6 & 0.09 & $\cdots$ & 0.07 \\
\hline 211916756 & 3315 & 228 & 0.42 & 0.08 & -2.06 & 0.22 & 0.26 & 0.13 \\
\hline 212002525 & 3380 & 203 & 0.22 & 0.03 & -2.46 & 0.17 & $\ldots$ & 0.1 \\
\hline 211901114 & 3389 & 135 & 0.4 & 0.03 & -1.9 & 0.11 & 0.37 & 0.12 \\
\hline 211428897 & 3393 & 101 & 0.38 & 0.03 & -1.93 & 0.07 & -0.11 & 0.04 \\
\hline $212154564^{\dagger}$ & 3429 & 117 & 0.33 & 0.03 & -1.71 & 0.11 & 0.11 & 0.1 \\
\hline $210750726^{\dagger}$ & 3526 & 90 & 0.5 & 0.03 & -1.38 & 0.08 & 0.07 & 0.07 \\
\hline $210838726^{\dagger}$ & 3574 & 86 & 0.52 & 0.02 & -1.48 & 0.08 & 0.03 & 0.06 \\
\hline $211305568^{\dagger}$ & 3618 & 130 & 0.53 & 0.05 & -1.41 & 0.11 & -0.004 & 0.07 \\
\hline 210495066 & 3621 & 90 & 0.5 & 0.02 & -1.27 & 0.09 & 0.05 & 0.07 \\
\hline 211843564 & 3681 & 112 & 0.54 & 0.02 & -1.45 & 0.16 & 0.48 & 0.13 \\
\hline $211969807^{\dagger}$ & 3720 & 140 & 0.49 & 0.02 & -1.3 & 0.14 & 0.5 & 0.12 \\
\hline $201617985^{\dagger}$ & 3726 & 148 & 0.52 & 0.03 & -1.35 & 0.16 & 0.17 & 0.13 \\
\hline 211831378 & 3737 & 99 & 0.54 & 0.02 & -1.3 & 0.13 & 0.26 & 0.12 \\
\hline $211924657^{\dagger}$ & 3766 & 208 & 0.68 & 0.16 & -1.91 & 0.16 & 0.1 & 0.1 \\
\hline 211357309 & 3778 & 99 & 0.47 & 0.03 & -1.47 & 0.05 & -0.03 & 0.04 \\
\hline $211509553^{\dagger}$ & 3786 & 129 & 0.49 & 0.03 & -1.21 & 0.12 & -0.18 & 0.13 \\
\hline $212006344^{\dagger}$ & 3837 & 75 & 0.58 & 0.027 & -1.15 & 0.06 & $\cdots$ & 0.03 \\
\hline $211331236^{\dagger}$ & 3847 & 124 & 0.51 & 0.04 & -1.37 & 0.10 & -0.06 & 0.07 \\
\hline $211799258^{\dagger}$ & 3857 & 224 & 0.38 & 0.03 & -1.72 & 0.12 & 0.26 & 0.11 \\
\hline 201833600 & 3911 & 148 & 0.57 & 0.04 & -0.99 & 0.27 & 0.32 & 0.2 \\
\hline $211822797^{\dagger}$ & 4004 & 103 & 0.56 & 0.02 & -1.16 & 0.11 & 0.51 & 0.07 \\
\hline $210508766^{\dagger}$ & 4058 & 322 & 0.72 & 0.09 & -1.69 & 0.67 & $\ldots$ & 0.33 \\
\hline 211336288 & 4076 & 106 & 0.57 & 0.02 & -1.4 & 0.12 & -0.39 & 0.08 \\
\hline $211762841^{\dagger}$ & 4105 & 127 & 0.83 & 0.07 & -1.28 & 0.17 & 0.19 & 0.1 \\
\hline $201635569^{\dagger}$ & 4174 & 153 & 0.59 & 0.02 & -0.67 & 0.19 & -0.33 & 0.2 \\
\hline 202071401 & 4177 & 113 & 0.71 & 0.04 & -0.73 & 0.10 & -0.49 & 0.07 \\
\hline $210968143^{\dagger}$ & 4187 & 98 & 0.66 & 0.02 & -1.02 & 0.09 & 0.16 & 0.05 \\
\hline
\end{tabular}

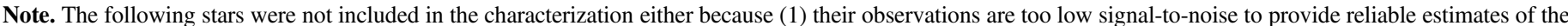

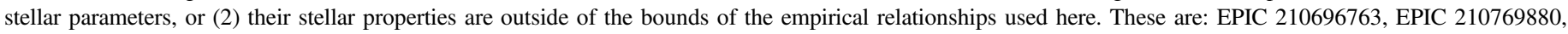

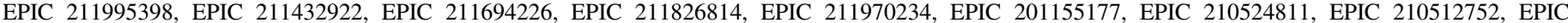

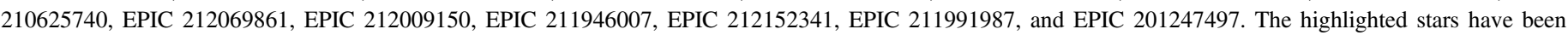
previously characterized in Dressing et al. (2017a).

that generates the light curve with the greatest photometric precision and quality.

Following the correction of systematics and removal of low-frequency variations in the light curves, we proceeded to reproduce the original light curves to assess the final transit and orbital parameters for our candidates. We estimated the systematic errors in our final light curve by fitting for them simultaneously with the transit parameters and low variability in the light curve. The systematics were modeled as a spline in arclength, or Kepler position in its roll, and the lowfrequency variability was modeled as a basis spline in time, with break points every 0.75 days. The light-curve transit parameters were modeled with the transit model from Mandel \& Agol (2002), while the fits to the transits were done using a Levenberg-Marquardt optimization (Markwardt 2009). We further analyze the light curves using the BATMAN Python package from Kreidberg (2015) to calculate the model transit light curves and thus estimate the final transit parameters and uncertainties. We assumed that all the planetary candidates were noninteracting. We included five parameters for each candidate: the time of the first transit, the period, inclination, the ratio of planet-to-star radius $\left(R_{p} / R_{\star}\right)$, and semimajor axis normalized to stellar radius $\left(a / R_{\star}\right)$. Finally, the transit parameters in the model were estimated using emcee (Foreman-Mackey et al. 2013), a Python package which simulates light curves using a Markov chain Monte Carlo algorithm. For a more thorough description of this procedure, see Mayo et al. (2018).

\subsection{Planet Properties}

For the calculation of the equilibrium temperature, we assume zero eccentricity and an albedo of zero. The median equilibrium temperature in the sample is $986 \mathrm{~K}$, while the majority of planets have radii between $2 R_{\oplus}$ and $4 R_{\oplus}$ rendering them Neptune-sized planets, consistent with the known planet size demographic of $\mathrm{M}$ dwarfs, most of which host subNeptune planets (Dressing \& Charbonneau 2013, 2015; Morton \& Swift 2014). All the derived planet parameters are 
Table 2

EWs and Masses for $K 2$ Cool Dwarfs

\begin{tabular}{|c|c|c|c|c|c|c|}
\hline EPIC & $\mathrm{Mg}(1.50 \mu \mathrm{m})$ & $\operatorname{Mg}(1.57 \mu \mathrm{m})$ & $\operatorname{Mg}(1.71 \mu \mathrm{m})$ & $\mathrm{Al}-\mathrm{a}(1.67 \mu \mathrm{m})$ & Al-b $(1.67 \mu \mathrm{m})$ & Mass $\left(M_{\odot}\right)$ \\
\hline 210564155 & 0.32 & 2.37 & 1.97 & 1.11 & 1.02 & $0.45^{\dagger}$ \\
\hline 211817229 & 0.74 & 0.70 & 0.97 & 0.31 & 0.75 & $0.45^{\dagger}$ \\
\hline 210659688 & 1.14 & 2.25 & 1.60 & 0.97 & 1.27 & $0.42^{\dagger}$ \\
\hline 212092746 & 2.26 & 0.53 & 1.44 & 1.02 & 2.37 & 0.18 \\
\hline 211839798 & 1.63 & 0.40 & 1.52 & 0.39 & 0.86 & $0.36^{\dagger}$ \\
\hline 210931967 & 2.25 & 2.00 & 2.29 & 0.94 & 1.70 & 0.41 \\
\hline 211077024 & 2.53 & 1.50 & 1.71 & 1.13 & 1.82 & 0.28 \\
\hline 201205469 & 3.99 & 3.76 & 3.30 & 1.13 & 2.79 & 0.61 \\
\hline 211916756 & 2.42 & 2.23 & 1.29 & 0.99 & 1.55 & 0.44 \\
\hline 212002525 & 1.74 & 1.06 & 0.80 & 1.04 & 1.00 & 0.44 \\
\hline 211901114 & 2.29 & 2.46 & 2.44 & 1.30 & 1.50 & 0.26 \\
\hline 211428897 & 2.44 & 2.20 & 1.68 & 1.05 & 1.43 & 0.36 \\
\hline 212154564 & 3.01 & 1.77 & 2.34 & 1.30 & 1.84 & 0.35 \\
\hline 210750726 & 4.32 & 3.26 & 2.61 & 1.22 & 2.19 & 0.51 \\
\hline 210838726 & 4.47 & 3.92 & 3.29 & 1.77 & 2.63 & 0.54 \\
\hline 211305568 & 4.13 & 2.98 & 2.48 & 0.95 & 1.76 & 0.58 \\
\hline 210495066 & 4.82 & 3.17 & 2.85 & 2.04 & 2.95 & 0.52 \\
\hline 211843564 & 4.77 & 4.07 & 3.40 & 2.01 & 2.63 & 0.44 \\
\hline 211969807 & 4.63 & 3.36 & 2.62 & 1.75 & 2.27 & 0.51 \\
\hline 201617985 & 4.89 & 3.76 & 3.27 & 1.52 & 2.18 & 0.55 \\
\hline 211831378 & 5.92 & 4.15 & 3.80 & 1.59 & 2.65 & 0.57 \\
\hline 211924657 & 3.47 & 2.82 & 0.93 & 0.69 & 1.19 & 0.53 \\
\hline 211357309 & 4.02 & 2.42 & 2.80 & 0.84 & 1.40 & 0.55 \\
\hline 211509553 & 5.24 & 3.40 & 3.12 & 1.39 & 2.13 & 0.52 \\
\hline 212006344 & 7.01 & 5.13 & 4.08 & 1.85 & 3.03 & 0.62 \\
\hline 211331236 & 5.14 & 3.42 & 3.50 & 1.22 & 1.83 & 0.63 \\
\hline 211799258 & 2.99 & 2.11 & 2.24 & 1.53 & 1.16 & 0.51 \\
\hline 201833600 & 7.67 & 4.38 & 4.09 & 1.54 & 2.86 & 0.60 \\
\hline 211822797 & 6.48 & 4.39 & 3.82 & 1.60 & 2.22 & 0.63 \\
\hline 210508766 & 7.68 & 6.94 & 4.91 & 2.19 & 3.10 & 0.65 \\
\hline 211336288 & 6.30 & 4.63 & 4.16 & 1.75 & 2.11 & 0.64 \\
\hline 211762841 & 7.34 & 5.37 & 4.35 & 1.07 & 2.04 & 0.60 \\
\hline 201635569 & 7.25 & 4.98 & 2.97 & 1.98 & 2.31 & 0.61 \\
\hline 202071401 & 7.64 & 5.29 & 3.56 & 1.26 & 2.14 & 0.70 \\
\hline 210968143 & 7.93 & 5.89 & 4.26 & 1.59 & 2.35 & 0.73 \\
\hline
\end{tabular}

Note. The highlighted masses were derived using the metric from Boyajian et al. (2012).

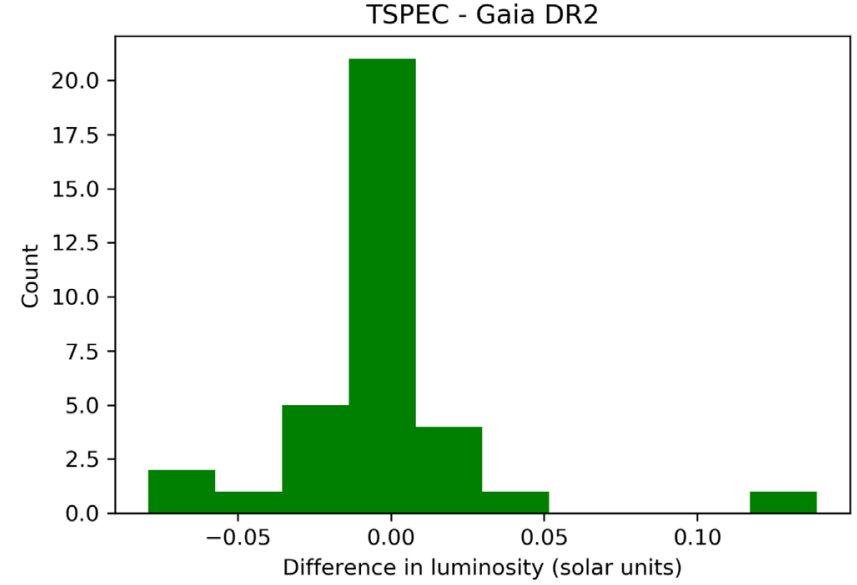

Figure 5. Difference between the distribution of the luminosity values (in solar units) of our stellar sample and those predicted by Gaia DR2. The underluminous outlier in the distribution is the early $M$ dwarf EPIC 201635569.

listed in Table 3. Figure 6 shows the distribution of sizes into four typical exoplanet size categories. We found two interesting outliers in the sample, EPIC 211995398 and EPIC 211509553, which show deep transits of $R_{p} / R \star=0.15$ and $R_{p} / R_{\star}=0.18$, respectively. This suggest that they may potentially harbor giant planets with sizes of $10.5 R_{\oplus}$ and $9.75 R_{\oplus}$. However, they are also large enough that they might be low-mass stars or brown dwarfs as well. We did not include the characterization of EPIC 211995398, because of the low signal-to-noise ratio of its observation. If confirmed to be true planets, these exoJupiters may become valuable laboratories for atmospheric characterization for future exoplanet missions. The same is true for planetary systems of bright and nearby hosts, which could be studied in more detail by the next generation of large space observatories, most notably the Transiting Exoplanet Survey Satellite (Ricker et al. 2014) and the James Webb Space Telescope (JWST).

As a first-order assessment of habitability, we considered the bulk composition of these planets and whether or not they reside in the habitable zone of their host stars (Hart 1979; Kasting et al. 1993; Shields et al. 2016). Rogers (2015) indicates that planets larger than $1.6 R_{\oplus}$ have densities too low to be rocky or terrestrial, and therefore are likely gaseous. Because we do not have measured masses for these planets, we can merely speculate from their inferred radii that probably only a small fraction of planets in our sample is rocky, based on Rogers (2015). To address the question of whether the planets 
Table 3

Planet Parameters for $K 2$ Cool Dwarfs

\begin{tabular}{|c|c|c|c|c|c|c|c|c|c|c|}
\hline EPIC & $R_{p} / R_{\star}$ & $R_{p}\left(R_{\oplus}\right)$ & $\sigma_{R_{p}}$ & $P$ (days) & $a(\mathrm{au})$ & $T_{\text {eq }}(\mathrm{K})$ & $\begin{array}{l}\text { VESPA False-positive } \\
\text { Probability }\end{array}$ & $\begin{array}{l}\text { Multiplicity } \\
\text { Boost }\end{array}$ & $\begin{array}{c}\text { Adopted False-positive } \\
\text { Probability }\end{array}$ & Designation \\
\hline 210495066 & 0.027 & 1.5 & 0.01 & 3.74 & 0.035 & 1038 & $6.05 \times 10^{-2}$ & $\mathrm{~N}$ & $6.05 \times 10^{-2}$ & Candidate \\
\hline 210508766 & 0.028 & 2.25 & 0.02 & 2.74 & 0.033 & 1000 & $8.44 \times 10^{-3}$ & $\mathrm{Y}$ & $3.37 \times 10^{-4}$ & Planet \\
\hline 210508766 & 0.034 & 2.69 & 0.02 & 9.99 & 0.078 & 650 & $4.90 \times 10^{-1}$ & $\mathrm{Y}$ & $1.96 \times 10^{-2}$ & Candidate \\
\hline 210659688 & 0.063 & 2.86 & 0.01 & 2.35 & 0.026 & 961 & $1.02 \times 10^{-1}$ & $\mathrm{~N}$ & $1.02 \times 10^{-1}$ & Candidate \\
\hline 210750726 & 0.044 & 2.42 & 0.01 & 4.61 & 0.04 & 946 & $1.00 \times 10^{-2}$ & $\mathrm{~N}$ & $1.00 \times 10^{-2}$ & Candidate \\
\hline 210838726 & 0.019 & 1.11 & 0.01 & 1.09 & 0.01 & 1472 & $1.85 \times 10^{-1}$ & $\mathrm{~N}$ & $1.85 \times 10^{-1}$ & Candidate \\
\hline 210968143 & 0.038 & 2.76 & 0.01 & 13.73 & 0.10 & 678 & $1.21 \times 10^{-3}$ & $\mathrm{Y}$ & $4.84 \times 10^{-5}$ & Planet \\
\hline 210968143 & 0.018 & 1.34 & 0.02 & 2.90 & 0.035 & 1139 & $2.31 \times 10^{-1}$ & $\mathrm{Y}$ & $9.24 \times 10^{-3}$ & Candidate \\
\hline 210931967 & 0.081 & 3.28 & 0.07 & 0.34 & 0.007 & 2072 & $9.95 \times 10^{-1}$ & $\mathrm{~N}$ & $9.95 \times 10^{-1}$ & Candidate \\
\hline 211077024 & 0.035 & 1.11 & 0.01 & 1.41 & 0.016 & 1351 & $1.12 \times 10^{-1}$ & $\mathrm{~N}$ & $1.12 \times 10^{-1}$ & Candidate \\
\hline 202071401 & 0.020 & 1.61 & 0.02 & 3.23 & 0.038 & 1186 & $7.85 \times 10^{-1}$ & $\mathrm{~N}$ & $7.85 \times 10^{-1}$ & Candidate \\
\hline 211305568 & 0.038 & 2.20 & 0.02 & 11.55 & 0.08 & 675 & & & & \\
\hline 211305568 & 0.015 & 0.87 & 0.02 & 0.19 & 0.005 & 2618 & $4.35 \times 10^{-1}$ & Y & $1.74 \times 10^{-2}$ & Candidate \\
\hline 211331236 & 0.037 & 2.07 & 0.01 & 1.29 & 0.02 & 1399 & $4.98 \times 10^{-3}$ & $\mathrm{Y}$ & $1.99 \times 10^{-4}$ & Planet \\
\hline 211331236 & 0.038 & 2.11 & 0.01 & 5.44 & 0.05 & 866 & $7.09 \times 10^{-2}$ & $\mathrm{Y}$ & $2.83 \times 10^{-3}$ & Candidate \\
\hline 211509553 & 0.180 & 9.65 & 0.00 & 20.35 & 0.118 & 600 & $1.09 \times 10^{-2}$ & $\mathrm{~N}$ & $1.09 \times 10^{-2}$ & Candidate \\
\hline 211762841 & 0.029 & 2.70 & 0.03 & 1.56 & 0.022 & 1351 & $4.40 \times 10^{-1}$ & $\mathrm{~N}$ & $4.40 \times 10^{-1}$ & Candidate \\
\hline 211817229 & 0.061 & 2.88 & 0.04 & 2.17 & 0.025 & 896 & $4.02 \times 10^{-1}$ & $\mathrm{~N}$ & $4.02 \times 10^{-1}$ & Candidate \\
\hline 211822797 & 0.033 & 2.03 & 0.01 & 21.16 & 0.128 & 581 & $1.79 \times 10^{-3}$ & $\mathrm{~N}$ & $1.79 \times 10^{-3}$ & Candidate \\
\hline 211843564 & 0.082 & 4.88 & 0.01 & 0.452 & 0.008 & 2064 & $4.27 \times 10^{-1}$ & $\mathrm{~N}$ & $4.27 \times 10^{-1}$ & Candidate \\
\hline 211901114 & 0.058 & 2.56 & 0.02 & 1.56 & 0.017 & 1308 & $3.18 \times 10^{-1}$ & $\mathrm{~N}$ & $3.18 \times 10^{-1}$ & Candidate \\
\hline 211916756 & 0.077 & 3.55 & 0.01 & 10.13 & 0.042 & 805 & $1.35 \times 10^{-2}$ & $\mathrm{~N}$ & $1.35 \times 10^{-2}$ & Candidate \\
\hline 201635569 & 0.107 & 6.95 & 0.02 & 8.36 & 0.068 & 900 & $5.22 \times 10^{-2}$ & $\mathrm{~N}$ & $5.22 \times 10^{-2}$ & Candidate \\
\hline 201833600 & 0.031 & 1.97 & 0.01 & 8.75 & 0.066 & 845 & $5.06 \times 10^{-2}$ & $\mathrm{~N}$ & $5.06 \times 10^{-2}$ & Candidate \\
\hline 201617985 & 0.031 & 1.77 & 0.02 & 7.28 & 0.06 & 807 & $9.92 \times 10^{-1}$ & $\mathrm{~N}$ & $9.92 \times 10^{-1}$ & Candidate \\
\hline 210564155 & 0.034 & 1.63 & 0.01 & 4.86 & 0.037 & 776 & $5.23 \times 10^{-2}$ & $\mathrm{~N}$ & $5.23 \times 10^{-2}$ & Candidate \\
\hline 212006344 & 0.020 & 1.27 & 0.01 & 2.21 & 0.028 & 1236 & $1.89 \times 10^{-3}$ & $\mathrm{~N}$ & $1.89 \times 10^{-3}$ & Candidate \\
\hline 212092746 & 0.043 & 0.66 & 0.00 & 0.56 & 0.007 & 1930 & $1.49 \times 10^{-1}$ & $\mathrm{~N}$ & $1.49 \times 10^{-1}$ & Candidate \\
\hline 211969807 & 0.038 & 2.07 & 0.03 & 1.97 & 0.02 & 1280 & 1.00 & $\mathrm{~N}$ & 1.00 & Candidate \\
\hline 211924657 & 0.054 & 4.03 & 0.03 & 2.64 & 0.03 & 990 & $3.27 \times 10^{-1}$ & $\mathrm{~N}$ & $3.27 \times 10^{-1}$ & Candidate \\
\hline 212154564 & 0.071 & 2.55 & 0.00 & 6.41 & 0.05 & 832 & $4.38 \times 10^{-4}$ & $\mathrm{~N}$ & $4.38 \times 10^{-4}$ & Planet \\
\hline 201205469 & 0.074 & 4.68 & 0.02 & 3.47 & 0.037 & 956 & $1.95 \times 10^{-1}$ & $\mathrm{~N}$ & $1.95 \times 10^{-1}$ & Candidate \\
\hline 211799258 & 0.259 & 10.75 & 0.01 & 19.53 & 0.11 & 535 & $8.22 \times 10^{-1}$ & $\mathrm{~N}$ & $8.22 \times 10^{-1}$ & Candidate \\
\hline
\end{tabular}

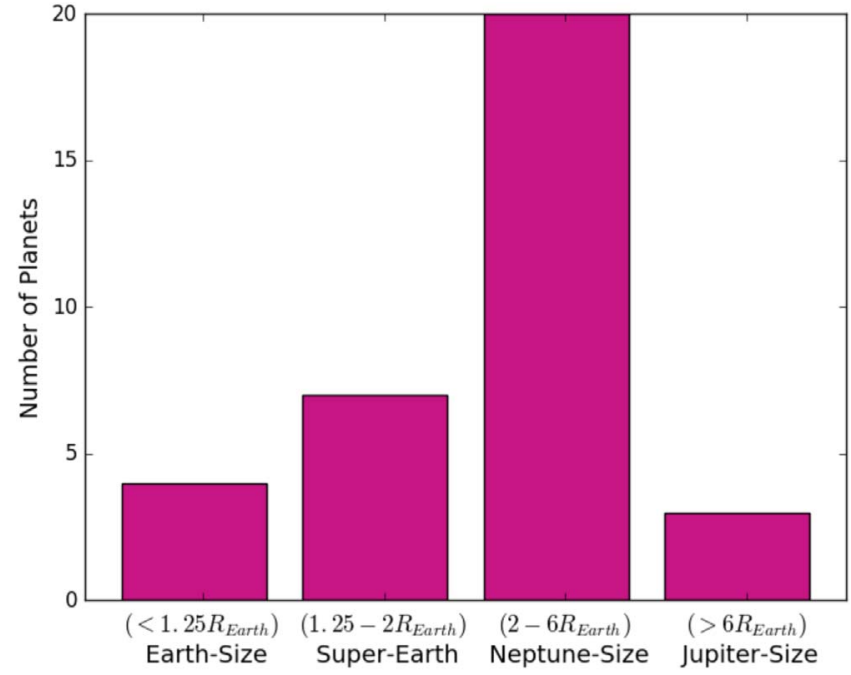

Figure 6. Planet-size distribution in four main categories: Earth size $\left(<1.25 R_{\oplus}\right)$, super-Earth size $\left(1.25-2 R_{\oplus}\right)$, Neptune size $\left(2-6 R_{\oplus}\right.$ and Jupiter size $\left(6-15 R_{\oplus}\right)$. The most numerous category is Neptune size, with 19 exoplanets, over half of the total sample size.

are in the habitable zone of their stars, we used the optimistic habitable zone boundaries for $\mathrm{M}$ dwarfs presented in Kopparapu et al. (2013). They determined that the habitable

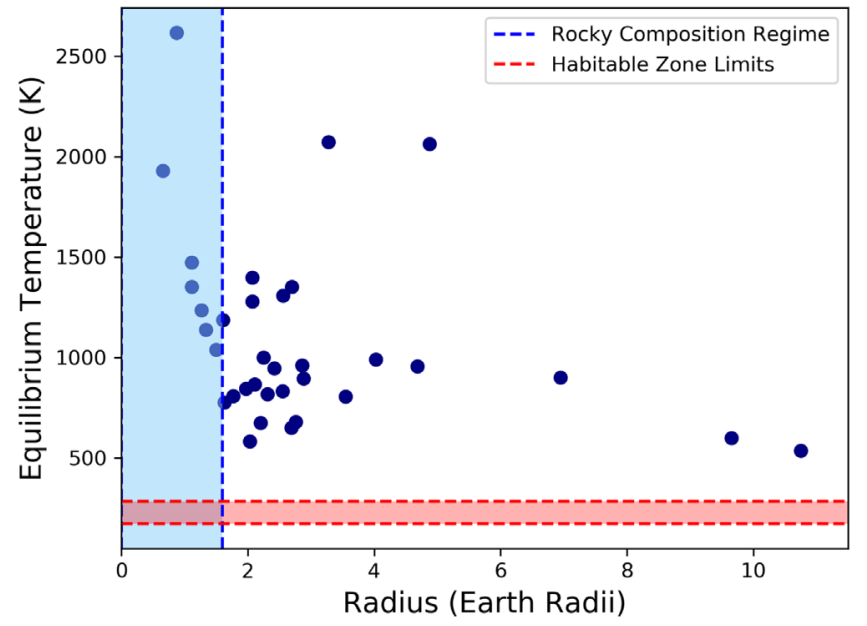

Figure 7. Distribution of sample of planets by equilibrium temperature vs. radius. The blue band defines the region within which planets have rocky compositions, based on Rogers (2015). The red band represents the range of temperatures where planets could be considered temperate for life based on Kopparapu et al. (2013). Under our definitions of habitability, potentially habitable planets would lie in the overlap of these two bands, the region in which they are both rocky and temperate.

zone for planets around typical $\mathrm{M}$ dwarfs is the circumstellar region between 0.09 and 0.24 au from the star (although these values are sensitive to temperature and vary for different 

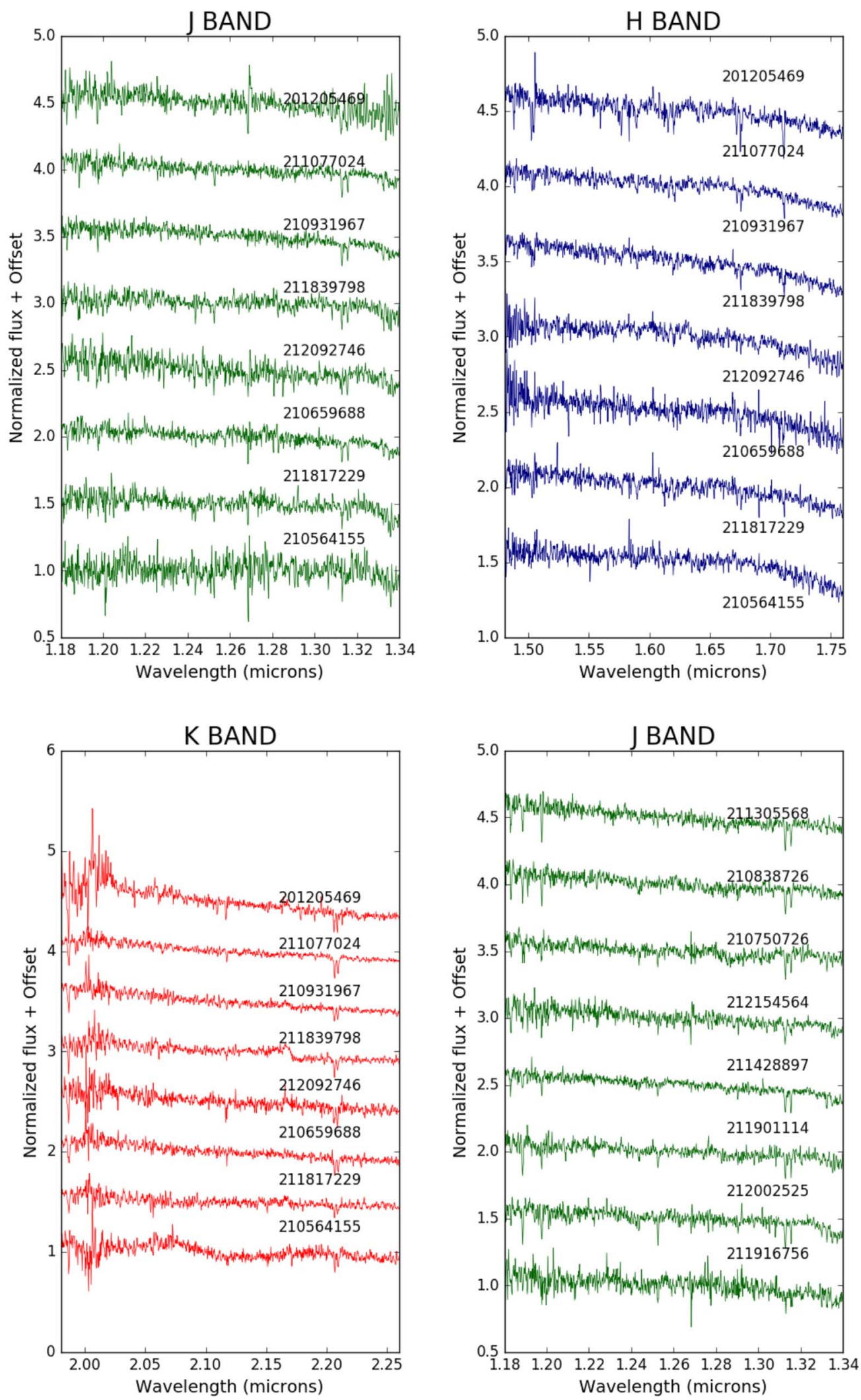

Figure 8. Multiple-band spectra of our cool dwarf sample. $J$ band (top left), $H$ band (top right) and $K$ band (bottom left). We are showing the spectra in the $H, J, K$ bands in order of decreasing temperature such that the hottest stars are on the top.

spectral types). Or equivalently, for planets to be considered within the habitable zone of $\mathrm{M}$ dwarfs, they must have equilibrium temperatures between $283 \mathrm{~K}$ (inner edge of habitable zone) and $171 \mathrm{~K}$ (outer edge), assuming an Earthlike, Bond albedo of 0.3. The planets in our sample, however, have very short-period orbits $(\bar{P}=5.9$ days $)$ and live well 

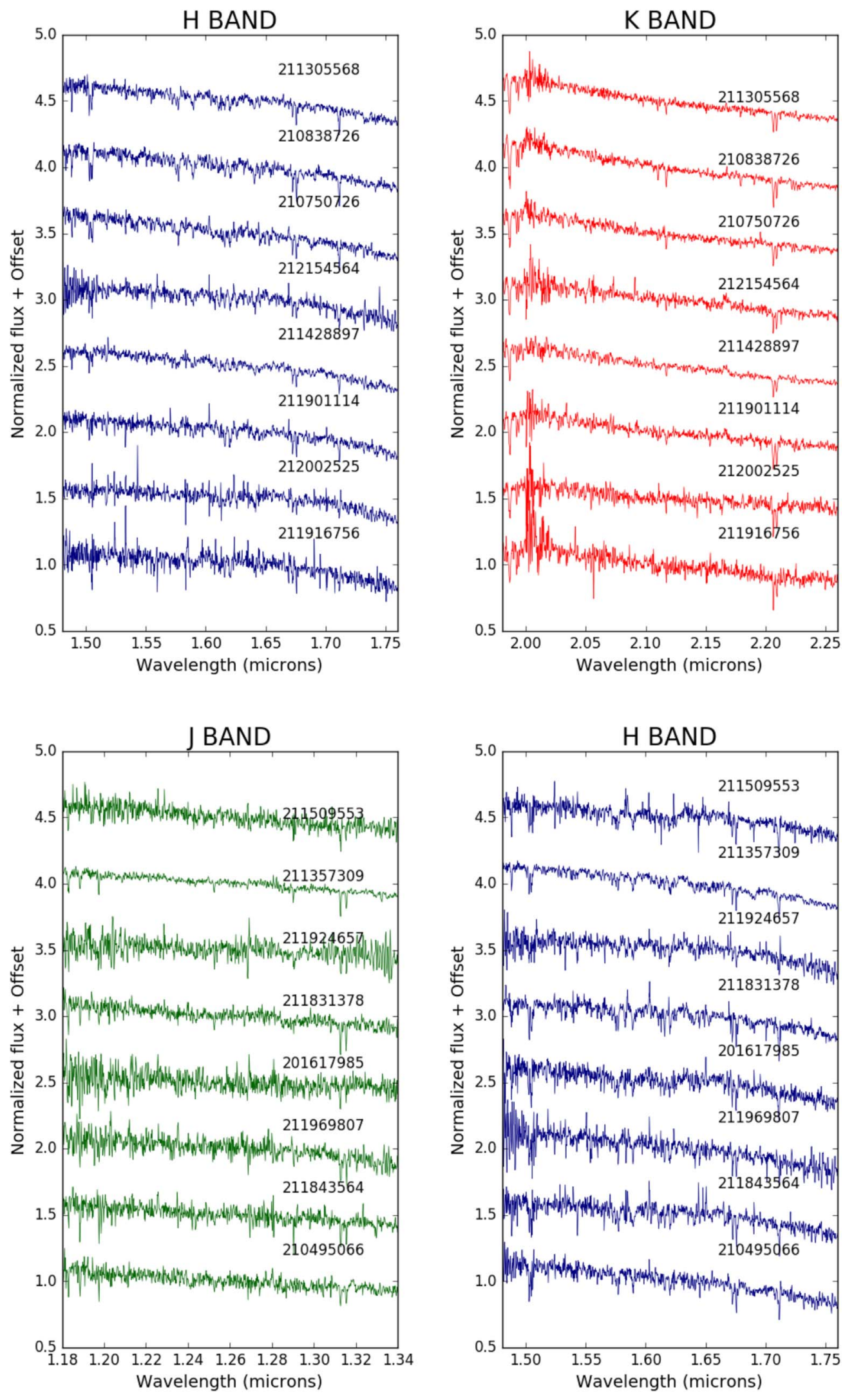

Figure 8. (Continued.)

inside the inner edge of the habitable zone. The proximity of these planets to their host stars render them too hot to be considered habitable, at least from the habitability metrics defined here. We conclude that there are no habitable planets in our sample, since there are none that are both rocky and on the habitable zone (see Figure 7). 

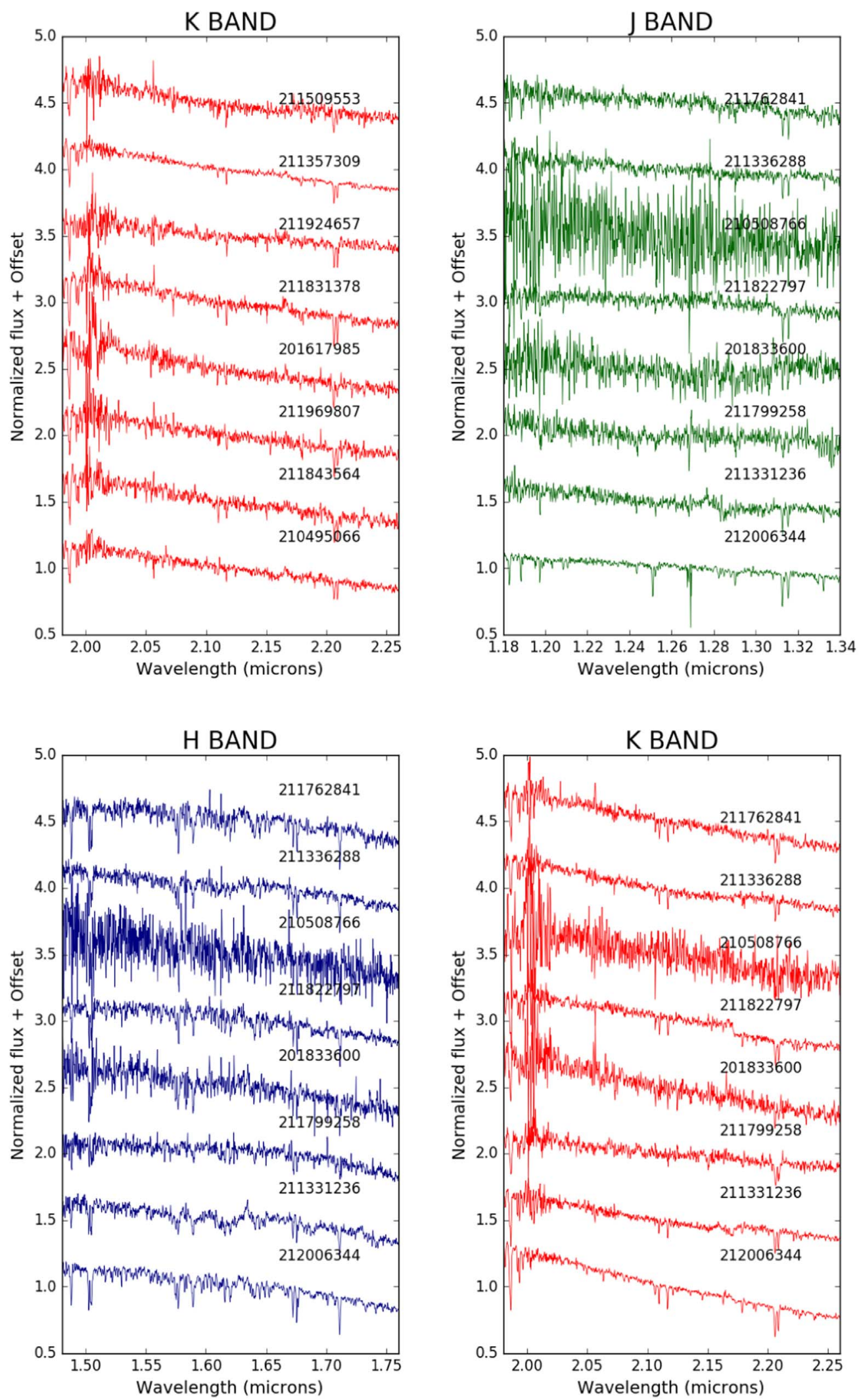

Figure 8. (Continued.)

\section{False Positives}

We assess the planetary nature of the candidate planet sample using vespa, a statistical validation framework developed by Morton $(2012,2015)$. This important tool allows the computation of the false-positive probability (FPP) of planet candidates by taking their transit and stellar parameters as an input. This provides a way to statistically confirm planets for which mass measurements from radial velocity are expensive or not feasible. 

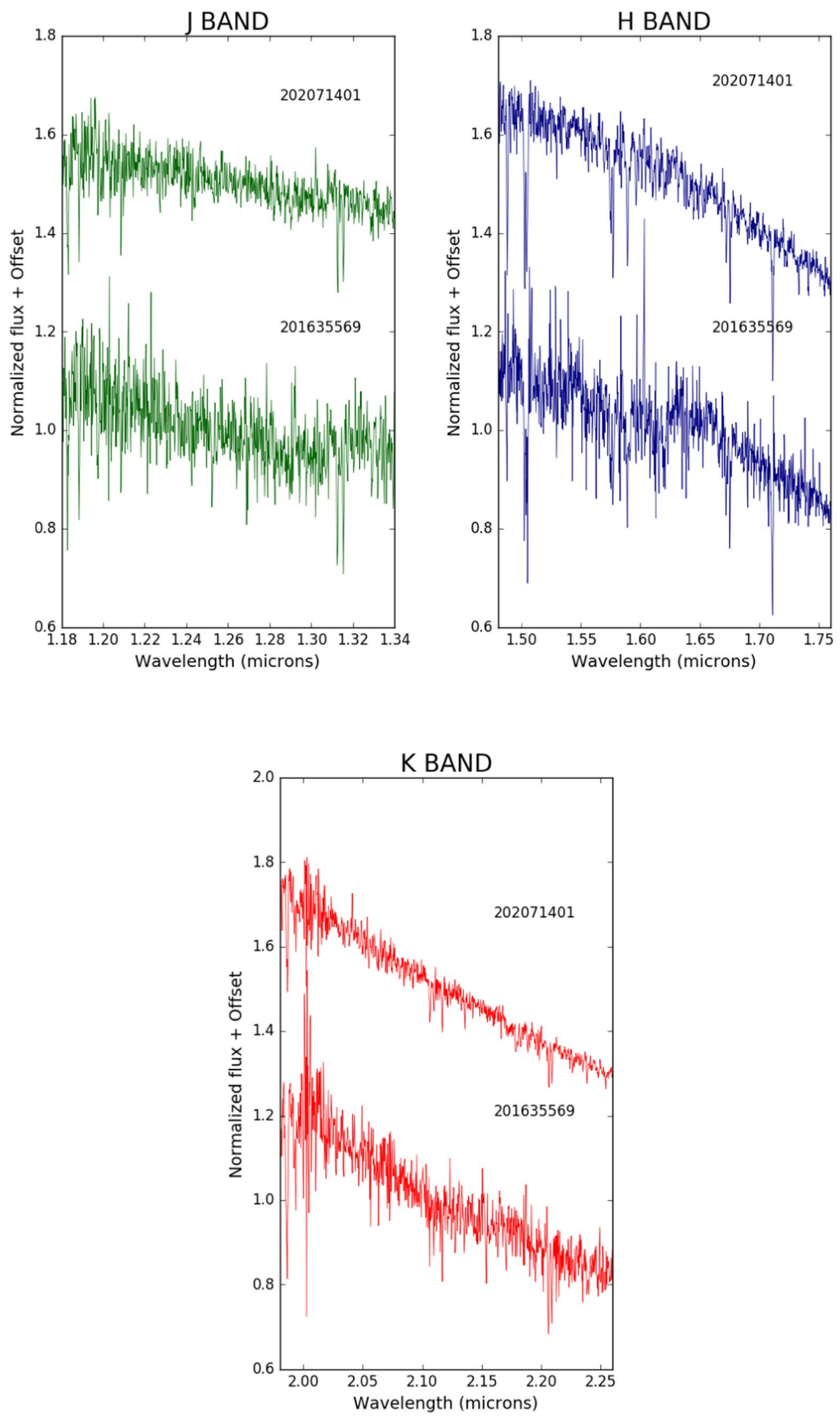

Figure 8. (Continued.) 
We supported our analysis of each planet candidate in our sample with observations from archival adaptive optics (AO) and speckle images in the Exoplanet Follow-up Observing Program for $K 2$ website (ExoFOP-K2). ${ }^{15}$ For EPIC 202071401, the available AO/speckle images from both Palomar and Keck II show a nearby companion at a separation of $<\sim 3^{\prime \prime}$, and even with the smallest aperture, we cannot rule out the possibility of a binary scenario. Moreover, we calculated a high FPP of $7.85 \times 10^{-1}$ for this object, so we classify it as a planet candidate. Similarly, images from the United Kingdom Infrared Telescope (UKIRT) in ExoFOP-K2 reveal a nearby, bound companion around EPIC 211305568. This early $\mathrm{M}$ dwarf has two candidates around it, one of which is classified as a planet candidate in Dressing et al. (2017b) due to their high FPP of 50\%-100\% from vespa and due to the nearby companion of the star. It is more probable for systems with two or more candidates to host true planets rather than multiple false-positive signals (Lissauer et al. 2012), so we can apply a boost to the probability of a planet scenario by reducing the FPP of an individual candidate by a factor of 25 for systems with two candidates, or 50 for systems of 3 or more. But even after applying this multiplicity boost to EPIC 211305568, the FPP of $1.74 \times 10^{-2}$ is too high to statistically confirm it. We also reject EPIC 211817229 because the $K 2$ light curve shows secondary eclipses, which is suggestive of a binary, although no companion is observed in the ExoFOP- $K 2$ images from UKIRT. The FPP for this candidate is also high, with $\mathrm{FPP}=4.02 \times 10^{-1}$.

EPIC 211509553 is reported in Pope et al. (2016) as a candidate with a large transit depth of $R_{p} / R \star=0.18$. Dressing et al. (2017b) identify it as a cool giant with a period of $P=20.3$ days and a radius of $R_{p}=10.8 \pm 0.6 R_{\oplus}$. Although this candidate meets their validation threshold of $<1 \%$ FPP, they cannot statistically confirm it due to the presence of a nearby companion. The available UKIRT and Gemini $8 \mathrm{~m}$ images show a clear stellar neighbor in the aperture for this star, and we compute an FPP of this candidate of $1.09 \times 10^{-2}$. We therefore classify it as a planet candidate, in agreement with the literature.

Pope et al. (2016) report EPIC 211995398 as a transiting planet candidate with a deep transit of $R_{p} / R_{\star}=0.19$ and period of $P=32.5$ days. A recent study found that $50 \%$ of Kepler giant exoplanet candidates are eclipsing binaries (Santerne et al. 2016), in contrast to a rate of $18 \%$ from previous study (Fressin et al. 2013). Fressin et al. (2013) had found that the Kepler (and K2) false-positive rate depends on planet size, peaking for giants $\left(6-22 R_{\oplus}\right)$ at $17.7 \%$. Santerne et al. (2016) also demonstrated that many of those false positives turn out to be brown dwarfs (Irwin et al. 2010; Johnson et al. 2011), with an occurrence rate of $\sim 2 \%$. Presently, very few hot Jupiters have been confirmed around M dwarfs (Johnson et al. 2012; Hartman et al. 2015; Bayliss et al. 2018). So although these large exoplanet candidates (EPIC 211509553 and EPIC 211995398) appear unique, it might be prudent to first rule out that they are false positives with spectroscopic follow-up.

We excluded 211839798 from the vespa run as we did not have transit information available for that target. In addition, vespa could not find the transits of the second candidate of 2113055568, so we do not have an FPP value for it. For the rest of the planet candidates for which the $K 2$ photometry and

\footnotetext{
15 https://exofop.ipac.caltech.edu/k2/
}

$\mathrm{AO} /$ speckle images were solid enough for vespa, we computed their FPP, applying the multiplicity boosts where applicable, and report them in Table 3. As in Mayo et al. (2018), we accept as statistically confirmed planets only those candidates with FPP $<0.001$, and classify anything above that cutoff threshold as a planet candidate. Out of the 33 total candidates, we classify 4 candidates as statistically validated planets and 29 as planet candidates. Figure 8 shows all of our reduced spectra for the M-dwarfs studied in this work.

\section{Conclusions}

In this study, we employ near-infrared spectra to derive the physical properties of a subset of M-dwarf exoplanets and their host stars, uncovered by $K 2$. We adopted a number of empirical calibrations for low-mass stars that relate the EWs of spectral features in the near-infrared to the stars' physical properties. We compared our EWs of a line sensitive to both radius and temperature to those from other publications and find that they are in good agreement. Our original sample of $K 2$ stars was contaminated by red giants or dwarfs of hotter classifications, and we discarded those from the characterization presented here. Additionally, we characterized the associated exoplanet candidates of the stellar sample using the inferred updated properties of their hosts. We specifically estimated the candidate planets' radius and temperature. Our planet sample is largely comprised of small planets, with 11 exoplanet candidates with $R_{p}<2 R_{\oplus}$ and 22 exoplanets (66\%) with $2 R_{\oplus}$ $<R_{p}<6 R_{\oplus}$. We assessed the habitability of these planets and determined that although some of them might be consistent with a rocky bulk composition, they are too highly radiated by their host stars to be in the habitable zone. Nevertheless, because the stars studied here are relatively bright targets $\left(\overline{K_{s}}=11.5\right)$, some of them could be suitable for follow-up characterization with JWST. In particular, we highlight two systems that are good for atmospheric characterization with the Hubble Space Telescope, Spitzer, or JWST, EPIC 211509553 (with $\mathrm{R}=9.65 R_{\oplus}$ and $R_{p} / R_{\star}=0.18$ ), which has been statistically validated in other publications as a cool giant and EPIC $211995398\left(\mathrm{R}=10.5 R_{\oplus}\right.$ and $\left.R_{p} / R_{\star}=0.15\right)$, which remains a candidate at present. Of our final sample of $35 \mathrm{M}$ dwarfs, 24 possess published characterization (Dressing et al. 2017a), while 11 are new to the literature. These 11 bring the total number of validated exoplanets to 318 from NASA's $K 2$ mission to date.

The authors thank the referee for the helpful comments that significantly improved the quality of this paper. The authors thank Juliette Becker for her assistance with the data reduction using Spextool. We also thank Nick Edwards, Yousef Lawrence, and Jonathan Swift (The Thacher School) for assisting with the data collection at Palomar Observatory. A.V.'s contribution to this study was performed in part under contract with the California Institute of Technology (Caltech)/ Jet Propulsion Laboratory (JPL) funded by NASA through the Sagan Fellowship Program executed by the NASA Exoplanet Science Institute. Work by B.T.M. was performed in part under contract with the Jet Propulsion Laboratory (JPL) funded by NASA through the Sagan Fellowship Program executed by the NASA Exoplanet Science Institute.

This work has made use of data from the European Space Agency (ESA) mission Gaia (www.cosmos.esa.int/ gaia), processed by the Gaia Data Processing and Analysis 
Consortium (DPAC; www.cosmos.esa.int/web/gaia/dpac/ consortium). Funding for the DPAC has been provided by national institutions, in particular the institutions participating in the Gaia Multilateral Agreement.

\section{ORCID iDs}

Andrew Mayo (i) https://orcid.org/0000-0002-7216-2135 Andrew Vanderburg (D) https://orcid.org/0000-00017246-5438

Benjamin T. Montet (1D https://orcid.org/0000-00017516-8308

Jessie L. Christiansen (10) https://orcid.org/0000-0002$8035-4778$

\section{References}

Anglada-Escudé, G., Amado, P. J., Barnes, J., et al. 2016, Natur, 536, 437 Ballard, S., \& Johnson, J. A. 2016, ApJ, 816, 66

Batalha, N. M., Rowe, J. F., Bryson, S. T., et al. 2013, ApJS, 204, 24 Bayliss, D., Gillen, E., Eigmüller, P., et al. 2018, MNRAS, 475, 4467 Benedict, G. F., Henry, T. J., Franz, O. G., et al. 2016, AJ, 152, 141 Bochanski, J. J., Hawley, S. L., Covey, K. R., et al. 2010, AJ, 139, 2679 Borucki, W. J., Koch, D., Basri, G., et al. 2010, Sci, 327, 977 Borucki, W. J., Koch, D. G., Basri, G., et al. 2011, ApJ, 728, 117 Boyajian, T. S., von Braun, K., van Belle, G., et al. 2012, ApJ, 757, 112 Brown, T. M., Latham, D. W., Everett, M. E., \& Esquerdo, G. A. 2011, AJ, 142,112

Burke, C. J., Bryson, S. T., Mullally, F., et al. 2014, ApJS, 210, 19

Cushing, M. C., Vacca, W. D., \& Rayner, J. T. 2004, PASP, 116, 362

Dawson, R. I., \& Murray-Clay, R. A. 2013, ApJL, 767, L24

Dittmann, J. A., Irwin, J. M., Charbonneau, D., et al. 2017, Natur, 544, 333

Dressing, C. D., Adams, E. R., Dupree, A. K., Kulesa, C., \& McCarthy, D. 2014, AJ, 148, 78

Dressing, C. D., \& Charbonneau, D. 2013, ApJ, 767, 95

Dressing, C. D., \& Charbonneau, D. 2015, ApJ, 807, 45

Dressing, C. D., Newton, E. R., Schlieder, J. E., et al. 2017a, ApJ, 836, 167

Dressing, C. D., Vanderburg, A., Schlieder, J. E., et al. 2017b, AJ, 154, 207

Fischer, D. A., \& Valenti, J. 2005, ApJ, 622, 1102

Foreman-Mackey, D., Hogg, D. W., Lang, D., \& Goodman, J. 2013, PASP, 125,306

Fressin, F., Torres, G., Charbonneau, D., et al. 2013, ApJ, 766, 81

Fulton, B. J., Petigura, E. A., Howard, A. W., et al. 2017, AJ, 154, 109

Gaia Collaboration, Brown, A. G. A., Vallenari, A., et al. 2018, A\&A, 616, A1

Gaia Collaboration, Prusti, T., de Bruijne, J. H. J., et al. 2016, A\&A, 595, A1

Gillon, M., Triaud, A. H. M. J., Demory, B.-O., et al. 2017, Natur, 542, 456 Hart, M. H. 1979, Icar, 37, 351

Hartman, J. D., Bayliss, D., Brahm, R., et al. 2015, AJ, 149, 166

Hirano, T., Dai, F. Gandolfi, D., et al. 2018, AJ, 155, 127

Howard, A. W., Marcy, G. W., Bryson, S. T., et al. 2012, ApJS, 201, 15

Howell, S. B., Sobeck, C., Haas, M., et al. 2014, PASP, 126, 398

Huber, D., Bryson, S. T., Haas, M. R., et al. 2016, ApJS, 224, 2
Huber, D., Kepler Eclipsing Binary Working Group, \& Asteroseismology Working Group 2013, BAAS, 45, 301.03

Irwin, J., Buchhave, L., Berta, Z. K., et al. 2010, ApJ, 718, 1353

Johnson, J. A., \& Apps, K. 2009, ApJ, 699, 933

Johnson, J. A., Apps, K., Gazak, J. Z., et al. 2011, ApJ, 730, 79

Johnson, J. A., Gazak, J. Z., Apps, K., et al. 2012, AJ, 143, 111

Jordi, C., Gebran, M., Carrasco, J. M., et al. 2010, A\&A, 523, A48

Kasting, J. F., Whitmire, D. P., \& Reynolds, R. T. 1993, Icar, 101, 108

Kopparapu, R. K., Ramirez, R., Kasting, J. F., et al. 2013, ApJ, 765, 131

Kreidberg, L. 2015, PASP, 127, 1161

Lissauer, J. J., Marcy, G. W., Rowe, J. F., et al. 2012, ApJ, 750, 112

Mandel, K., \& Agol, E. 2002, ApJL, 580, L171

Mann, A. W., Brewer, J. M., Gaidos, E., Lépine, S., \& Hilton, E. J. 2013a, AJ, 145,52

Mann, A. W., Gaidos, E., \& Ansdell, M. 2013b, ApJ, 779, 188

Mann, A. W., Gaidos, E., Lépine, S., \& Hilton, E. J. 2012, ApJ, 753, 90

Markwardt, C. B. 2009, in ASP Conf. Ser. 411, Astronomical Data Analysis Software and Systems XVIII, ed. D. A. Bohlender, D. Durand, \& P. Dowler (San Fransisco, CA: ASP), 251

Martinez, A. O., Crossfield, I. J. M., Schlieder, J. E., et al. 2017, ApJ, 837, 72

Mayo, A. W., Vanderburg, A., Latham, D. W., et al. 2018, AJ, 155, 136

Morton, T. D. 2012, ApJ, 761, 6

Morton, T. D. 2015, VESPA: False positive probabilities calculator, Astrophysics Source Code Library, ascl:1503.011

Morton, T. D., \& Swift, J. 2014, ApJ, 791, 10

Muirhead, P. S., Dressing, C. D., Mann, A. W., et al. 2018, AJ, 155, 180

Mulders, G. D., Pascucci, I., \& Apai, D. 2014, AAS/DPS Meeting Abstracts, 46, 301.09

Mulders, G. D., Pascucci, I., \& Apai, D. 2015, ApJ, 814, 130

Newton, E. R., Charbonneau, D., Irwin, J., et al. 2014, AJ, 147, 20

Newton, E. R., Charbonneau, D., Irwin, J., \& Mann, A. W. 2015, ApJ, 800, 85

Pope, B. J. S., Parviainen, H., \& Aigrain, S. 2016, MNRAS, 461, 3399

Reid, I. N., \& Hawley, S. L. (ed.) 2005, New Light on Dark Stars Red Dwarfs, Low-Mass Stars, Brown Stars (Berlin: Springer)

Ricker, G. R., Winn, J. N., Vanderspek, R., et al. 2014, Proc. SPIE, 9143, 914320

Rogers, L. A. 2015, ApJ, 801, 41

Rojas-Ayala, B., Covey, K. R., Muirhead, P. S., \& Lloyd, J. P. 2012, ApJ, 748, 93

Santerne, A., Moutou, C., Tsantaki, M., et al. 2016, A\&A, 587, A64

Santos, N. C., Israelian, G., \& Mayor, M. 2004, A\&A, 415, 1153

Shields, A. L., Ballard, S., \& Johnson, J. A. 2016, PhR, 663, 1

Sullivan, P. W., Winn, J. N., Berta-Thompson, Z. K., et al. 2015, ApJ, 809, 77

Terrien, R. C., Mahadevan, S., Bender, C. F., et al. 2012, ApJL, 747, L38

Torres, G., Fischer, D. A., Sozzetti, A., et al. 2011, AAS/ESS Meeting, 2, 19.03

Vacca, W. D., Cushing, M. C., \& Rayner, J. T. 2003, PASP, 115, 389

Van Eylen, V., Agentoft, C., Lundkvist, M. S., et al. 2018, MNRAS, 479, 4786

Vanderburg, A., Bieryla, A., Duev, D. A., et al. 2016, ApJL, 829, L9

Vanderburg, A., \& Johnson, J. A. 2014, PASP, 126, 948

Weiss, L. M., Marcy, G. W., Rowe, J., et al. 2013, AAS Meeting Abstracts, $221,407.05$

Wilson, J. C., Henderson, C. P., Herter, T. L., et al. 2004, Proc. SPIE, 5492,1295

Wolfgang, A., Rogers, L. A., \& Ford, E. B. 2016, ApJ, 825, 19 